\title{
Evidence for synchrotron bubbles from GRS 1915+105
}

\author{
C. H. Ishwara-Chandra ${ }^{1}$, J. S. Yadav ${ }^{2}$, and A. Pramesh Rao ${ }^{3}$ \\ 1 Space Astronomy and Instrumentation Division, ISRO Satellite Center, Bangalore 560 017, India \\ 2 Tata Institute of Fundamental Research, Homi Bhaba Road, Mumbai 400 005, India \\ 3 National Center for Radio Astrophysics, Post Bag No. 3, Ganeshkhind, Pune 411 007, India
}

Received 18 February 2002 / Accepted 12 April 2002

\begin{abstract}
We present GMRT observations of the Galactic microquasar GRS $1915+105$ at $1.28 \mathrm{GHz}$ for 8 days from 2001 June 18 to July 1 . We have seen several isolated radio flares of varying magnitudes $(20-50 \mathrm{mJy})$ and durations (6-35 min) and we model them as due to adiabatically expanding synchrotron emitting clouds (synchrotron bubbles) ejected from the accretion disk. By applying this model, we provide a new method to estimate the electron power-law index $p$, hence the spectral index, from single frequency radio observations. This method does not require correction for the optical time delay effects which may be important in the case of optically thick radio emission. Using our estimated value of $p$ and simultaneous multiwavelength data from literature, we have calculated the time of ejection of the synchrotron plasma and the time delays at different observed frequencies. Our estimates are in good agreement with the observed time delays.
\end{abstract}

Key words. stars: individual: GRS $1915+105$ - radio continuum: stars - X-rays: binaries

\section{Introduction}

The Galactic X-ray transient source GRS $1915+105$ was discovered in 1992 by the GRANAT satellite (Castro-Tirado et al. 1992). It has now been established as a black hole binary with the black hole mass of $14 \pm 4 M_{\odot}$ and the companion K-M III star of mass $1.2 \pm 0.2 M_{\odot}$ (Greiner et al. 2001). The source was shown to possess relativistic outflows during its radio outbursts (Mirabel \& Rodríguez 1994). Extensive monitoring of GRS $1915+105$ in the radio and X-ray band showed that the overall radio emission is correlated with its X-ray properties (e.g. Harmon et al. 1997). The radio emission can be broadly classified into three classes; (i) the relativistic superluminal radio jets of flux density $\sim 1$ Jy with decay time-scales of several days (Fender et al. 1999), (ii) the baby jets of 20-40 min durations with flux density of 20-200 mJy both in infrared (IR) and radio (Pooley \& Fender 1997; Eikenberry et al. 1998) and (iii) the plateau state with persistent radio emission of 20-100 mJy for extended durations (Muno et al. 2001). In the case of superluminal jets, the radio emission has steep spectra and are observed at large distances (400-5000 AU) from the accretion disk (Fender et al. 1999; Dhawan et al. 2000). The radio emission at this distance is believed to be decoupled from the accretion disk (Yadav 2001; Muno et al. 2001). In contrast, the other two classes of radio emission has flat spectra and occur close to

Send offprint requests to: C. H. Ishwara-Chandra,

e-mail: ishwar@ncra.tifr.res.in the accretion disk (within a few tens of AU). Even though superluminal jets and baby jets are differentiated by their spectra, decay time scales, and the distance (from the accretion disk) where the emission occurs, there is evidence for ejection of significant amount of relativistic material even during the baby jets (Fender et al. 1999). The radio emission is believed to be due to synchrotron emission from relativistic electrons and the dominant decay mechanism is the adiabatic expansion losses (Mirabel et al. 1998, hereinafter M98). Feroci et al. (1999) have studied an isolated radio flare $(\sim 60 \mathrm{mJy}$ at $15 \mathrm{GHz})$ in X-ray and radio and present strong evidence for mass ejection (QPO disappears during the radio outburst). Coordinated multiwavelength studies have indicated strong disk-jet connection (M98; Eikenberry et al. 1998; Yadav 2001).

The time of the ejection of the radio emitting synchrotron plasma from the accretion disk is an important parameter to understand the disk-jet connection. Another important parameter is the power-law index $(p)$ of the electron energy distribution in the plasma which is related to the delay time. Even though simultaneous multi-wavelength radio observations provide $p$, in the case of optically thick radio emission it is affected by the optical depth time delay effects and may introduce large error in the estimate of time of ejection, particularly in the case of periodic emission like IR and radio baby jets.

In this Letter we provide a new method, by applying the adiabatic expansion model to baby jets to estimate $p$ from single frequency radio observations. This method 
has the unique advantage in the sense that it surpasses the optical depth effects. We apply this method to isolated baby jets seen in the radio observations of GRS 1915+105 with the Giant Meterwave Radio Telescope (GMRT) at $1.28 \mathrm{GHz}$ and estimate $p$ and the time of ejection of the synchrotron bubble. Using this $p$ and the multiwavelength data from literature we also calculate the time delays at different observed frequencies and the time at which the synchrotron bubble is ejected. In Sect. 2 we present the observational details and basic results. The adiabatic expansion model is given in Sect. 3. Discussions and conclusions are presented in Sect. 4.

\section{Observations and results}

The radio observations were carried out at $1.28 \mathrm{GHz}$ with a bandwidth of $16 \mathrm{MHz}$ using the GMRT (Swarup et al. 1991) on 2001 June 18, 22, 23, 27, 28, 29, 30 and July 1. The flux density scale is set by observing the primary calibrator $3 \mathrm{C} 286$ or $3 \mathrm{C} 48$. A phase calibrator was observed before and after a 45 min scan on GRS 1915+105. The integration time was $32 \mathrm{~s}$. The data recorded from GMRT has been converted to FITS and was analysed using Astronomical Image Processing System (AIPS). The lightcurve produced at $5 \mathrm{~min}$ interval is shown in Fig. 1 (top panel). GRS $1915+105$ exhibited significant radio emission on all days, except on June 27. On June 18, the source was quite steady with the flux density $\sim 10 \mathrm{mJy}$. However, on June 22 the source became bright ( 50 mJy) and showed rapid drop in the flux density by a factor of two and slowly recovered to about the same level. On June 23, the flux density was steady at about $30 \mathrm{mJy}$. The source became very weak in radio $(\sim 5 \mathrm{mJy})$ on June 27. However on 28 , the flux density was $\sim 5 \mathrm{mJy}$ at about UTC 17 hour (beginning of observation) and reached gradually to $50 \mathrm{mJy}$ at UTC 24 hour. When the observations resumed on 29 , the source was "caught" at $70 \mathrm{mJy}$ at UTC 16 hour, and the flux started decaying slowly to the value of 10 mJy at UTC 24 hour. The source was relatively faint on June 30 and July 1.

We have checked the radio data at shorter binning time of $1 \mathrm{~min}$, which showed clear short term variations. Minor flares of amplitude upto $50 \mathrm{mJy}$ have been seen on June 28, 29, 30 and July 1, which are typical of baby jets (Fig. 3 and Table 1 ). The X-ray hardness ratio (5$12 \mathrm{keV} / 1.5-5 \mathrm{keV})$ from RXTE/ASM shown in the bottom panel of Fig. 1 suggests that overall GRS 1915+105 remained in the low hard state.

\section{Adiabatic expansion model}

We propose that the minor flares (baby jets) seen in the GMRT observations are due to adiabatically expanding synchrotron emitting bubbles. Hjellming \& Johnston (1988) present models for explaining the persistent radio emission from X-ray binaries by assuming adiabatic expansion dominated by lateral motions. For flaring events,

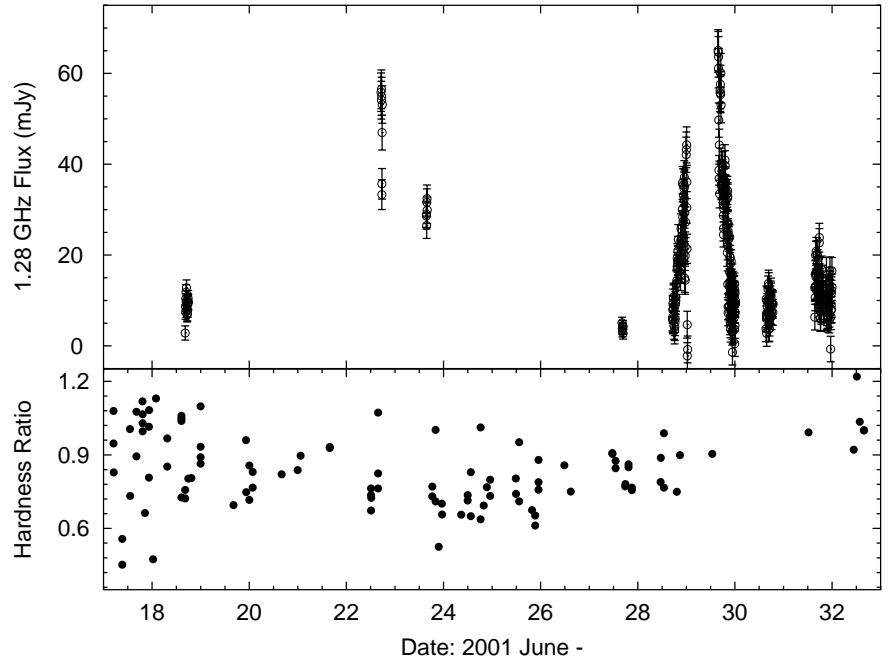

Fig. 1. Top panel: radio light curve from GMRT at $1.28 \mathrm{GHz}$, binned at $5 \mathrm{~min}$ interval. The observations on 2001 June 18, 22,23 and 27 is for $\sim 30-60 \mathrm{~min}$, while rest of the days are of $\sim 8-10$ hours. Bottom panel: RXTE/ASM hardness ratio (5-12 keV/1.5-5 keV) for the same duration.

they consider spherical bubbles of relativistic plasma expanding under its pressure. We consider this simple adiabatically expanding sphere model (three dimensional expansion) for the baby jets seen in the GMRT observations to understand their overall radio behavior. We assume that the expansion is uniform and the transition time from optically thick to thin state is negligible.

Consider a spherical cloud of relativistic electrons with the energy distribution $N(E) \mathrm{d} E \propto E^{-p} \mathrm{~d} E$, emitting synchrotron radiation. If this cloud is expanding adiabatically, the observed flux density as a function of time will be (van der Laan 1966; Hjellming \& Johnston 1988)

$S_{\mathrm{p}} / S_{\mathrm{s}}=\left(r_{\mathrm{p}} / r_{\mathrm{s}}\right)^{3}$

under optically thick conditions, and

$S_{\mathrm{e}} / S_{\mathrm{p}}=\left(r_{\mathrm{e}} / r_{\mathrm{p}}\right)^{-2 p}$

under optically thin conditions. Here $S_{\mathrm{s}}, S_{\mathrm{p}}$ and $S_{\mathrm{e}}$ are the observed flux densities at the start, peak and the end of the flare and $r_{\mathrm{s}}, r_{\mathrm{p}}$ and $r_{\mathrm{e}}$ are the corresponding radii of the bubble. $p$ is the power-law index of electron energy distribution. We define the time sequence of synchrotron bubble as in Fig. 2. $t_{\mathrm{s}}$ is the time taken for the bubble to attain the flux density $S_{\mathrm{s}}, t_{\mathrm{p}}$ is the time taken since $t_{\mathrm{s}}$ for the flux density to reach its peak value $S_{\mathrm{p}}$ (expansion under optically thick conditions) and $t_{\mathrm{e}}$ is the time taken since $t_{\mathrm{p}}$ by the bubble to decay to the flux level of $S_{\mathrm{e}}$ (expansion under optically thin conditions). Assuming uniform expansion, the profiles will be

$S_{\mathrm{p}}=S_{\mathrm{s}}\left[1+\left(t_{\mathrm{p}} / t_{\mathrm{s}}\right)\right]^{3}$

for the rising part of the light curve and

$S_{\mathrm{e}}=S_{\mathrm{p}}\left\{1+\left[t_{\mathrm{e}} /\left(t_{\mathrm{p}}+t_{\mathrm{s}}\right)\right]\right\}^{-2 p}$ 


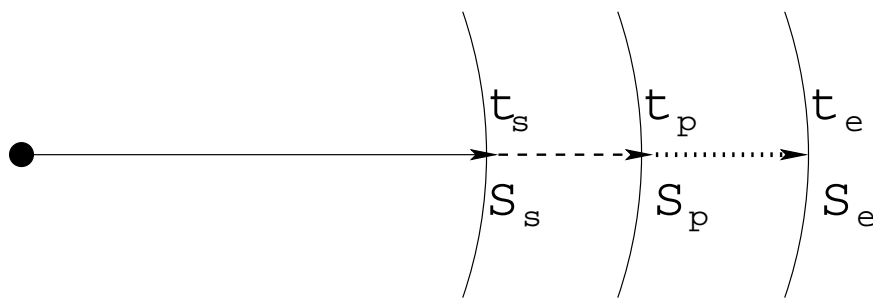

Fig. 2. Illustration of the time markers during the adiabatic expansion. The point on the left marks the ejection time.

for the decay part. An interesting case is when the synchrotron bubble is isolated, where the rise, peak and decay of a single bubble is observed. The adiabatic Eqs. (1) and (2) can be solved to obtain $p$

$p=\frac{1}{2} \frac{\log \left(S_{\mathrm{p}} / S_{\mathrm{e}}\right)}{\log \left\{1+\left(t_{\mathrm{e}} / t_{\mathrm{p}}\right)\left[1-\left(S_{\mathrm{p}} / S_{\mathrm{s}}\right)^{-1 / 3}\right]\right\}}$.

Thus by measuring the rise and decay time from isolated flares, the electron power-law index $p$ can be obtained. For estimating $p$, we have considered only those flares where the rise and decay of single flare is clearly visible well above the noise level or steady emission. We fit the above equations (using the least square method) to such isolated flares seen in our data on 2001 June 28, 29, 30 and July 1. The best fit model for two such flares are given in Fig. 3. The estimated parameters are given in Table 1 . Column 1 gives the date of observation, Col. 2 gives the estimated time (UT) of birth of the synchrotron bubble (see Sect. 4 for details). The duration and peak flux density of the flares are given in Cols. 3 and 4 . The electron power-law index $(p)$ and the spectral index $(\alpha=(p-1) / 2)$ are given in Cols. 5 and 6.

\section{Discussion and conclusion}

We have presented the GMRT observations of the microquasar GRS $1915+105$ at $1.28 \mathrm{GHz}$. Several isolated minor flares which are typical of baby jets have been found. We have suggested that these flares are due to adiabatically expanding bubbles of synchrotron emitting plasma. By applying adiabatic expansion model we have provided a new method to estimate the electron power-law index $p$ from single frequency radio observations, which does not need correction for the optical depth time delay effects. The simultaneous multi-wavelength radio observations of GRS $1915+105$ reported by Fender et al. (2002) reveal short time large variations in the spectral index from optically thick $(\alpha \sim-0.2)^{1}$ to thin values $(\alpha \sim 0.7)$ which corresponds to $p$ of 2.4 in the optically thin regime. They observed the fast oscillations of about half an hour period on MJD 51751. The power-law index $p$ obtained from our observations (Table 1) are in agreement with the measured values of Fender et al. (2002) in the optically thin region. The simplest explanation to this is that at the peak of the oscillation, the contribution from the optically thick

\footnotetext{
${ }^{1}$ We define the radio spectral index $\alpha$ as $S_{\nu} \propto \nu^{-\alpha}$.
}
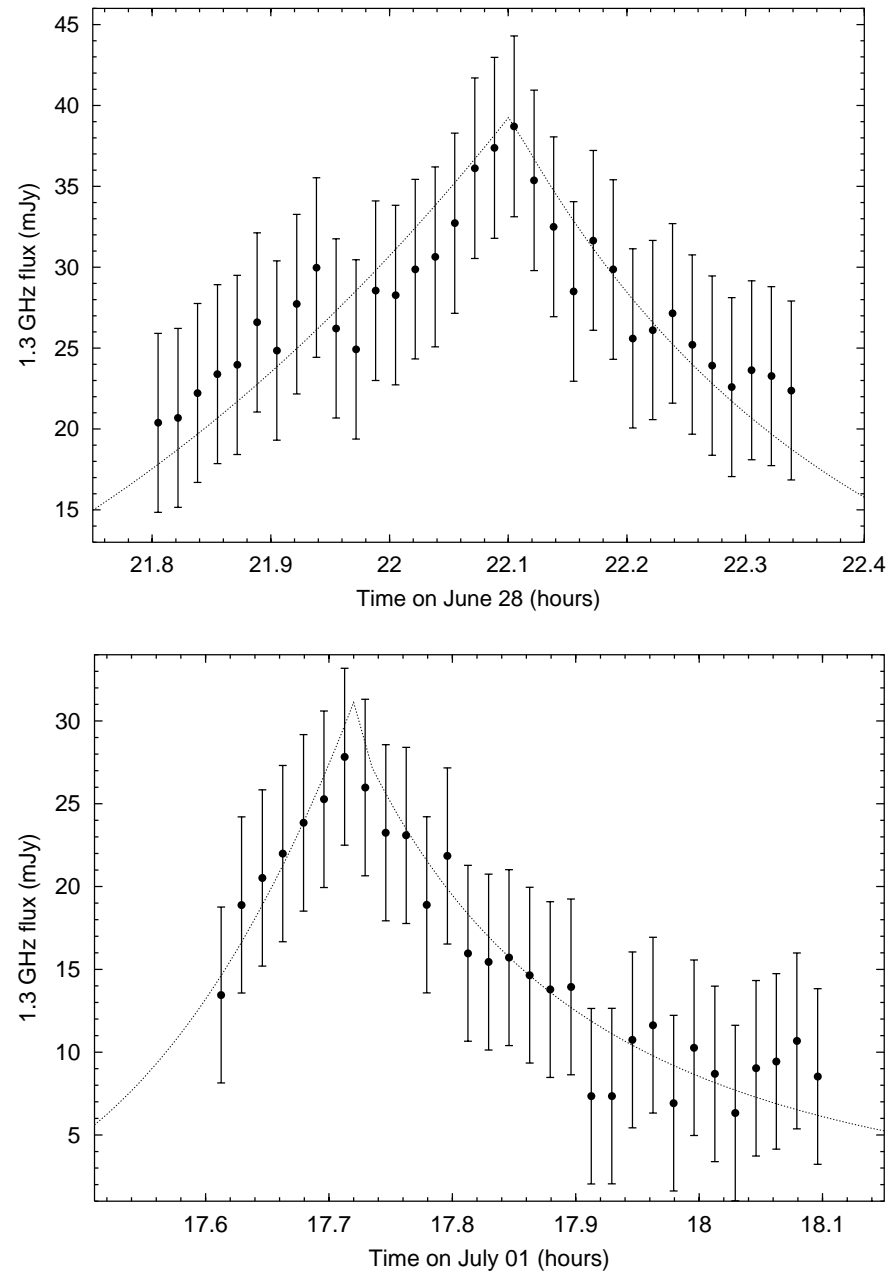

Fig. 3. Examples of isolated flares from GMRT observations. Top panel: the isolated flare seen on 2001 June 28. Bottom panel: one of the four minor isolated flares seen on July 1 . The data is binned at $1 \mathrm{~min}$ interval, the time is in UT. The dotted line is the fit to the Eqs. (3) and (4) for the rising part and for the optically thin decay respectively.

radio emission is large and hence the delay time correction becomes important. However, at low flux level the contribution from optically thick emission is reduced and the delay time correction is less important. This explanation for variations in $\alpha$ reported by Fender et al. (2002) suggests that intrinsically $p$ could be constant which is consistent with an adiabatically expanding synchrotron source with no additional acceleration. It is difficult to visualise change in $p$ over such a short duration of $\sim 15 \mathrm{~min}$.

We can calculate the time delays between emission at different wavelengths using this $p$. The time at which the emission at an wavelength $\lambda$ reaches its maximum is given by (van der Laan 1966)

$t_{\mathrm{m}, \lambda} \propto \lambda^{\frac{p+4}{4 p+6}}$.

We calculate the proportionality constant of Eq. (6) using our estimate of $p$ and the time delay of $0.25 \mathrm{~h}$ between $8.3 \mathrm{GHz}$ and $5 \mathrm{GHz}$ radio emission observed in GRS $1915+105$ by M98. The value of $t_{6 \mathrm{~cm}}$ estimated by 
Table 1. Estimated parameters for the synchrotron bubbles from GMRT Observations at $1.28 \mathrm{GHz}$.

\begin{tabular}{cccccc}
\hline \hline $\begin{array}{c}\text { Obsn } \\
\text { date }\end{array}$ & $\begin{array}{c}\text { Time of } \\
\text { ejection }\end{array}$ & $\begin{array}{c}\text { Dur } \\
(\min )\end{array}$ & $\begin{array}{c}\text { Peak } \\
\text { Flux }\end{array}$ & $p$ & $\alpha$ \\
\hline $06 / 28$ & 19.8126 & $\sim 30$ & 38.8 & $2.17 \pm 0.17$ & $0.58 \pm 0.09$ \\
$06 / 29$ & 14.0653 & $\sim 8$ & 51.3 & $1.25 \pm 0.12$ & $0.13 \pm 0.06$ \\
$06 / 30$ & 18.0620 & $\sim 30$ & 30.1 & $1.64 \pm 0.08$ & $0.32 \pm 0.04$ \\
$07 / 01$ & 15.5077 & $\sim 35$ & 27.9 & $1.36 \pm 0.07$ & $0.18 \pm 0.04$ \\
$07 / 01$ & 20.3278 & $\sim 12$ & 19.4 & $1.51 \pm 0.13$ & $0.25 \pm 0.06$ \\
$07 / 01$ & 21.5200 & $\sim 9$ & 20.5 & $1.12 \pm 0.11$ & $0.06 \pm 0.05$ \\
$07 / 01$ & 21.6818 & $\sim 6$ & 32.8 & $2.01 \pm 0.32$ & $0.50 \pm 0.16$ \\
\hline
\end{tabular}

us for May 15, 1997 observations of M98 is $~ 1.2 \mathrm{~h}$ (M98 gives $0.9 \mathrm{~h}$ assuming $p=0$ ) and the ejection of plasma occurred at $14.95-1.2=13.75 \mathrm{~h}$. Inspection of the Xray data at this time (Fig. 1 of M98) shows that it still falls in the gap in the X-ray data. We have calculated the time of formation of the synchrotron bubble for the flares observed by us which are given in Table 1 .

We give in Table 2 the observed time delays between different frequencies from simultaneous multiwavelength observations of GRS $1915+105$ reported in the literature. We have estimated the time delays between these frequencies using the weighted mean value of $p=1.46$ from our observations which are also given in Table 2. For observations on MJD 50675, the calculated time delay of $37 \mathrm{~s}$ for the IR emission is consistent with the observation that the spikes in X-ray coincide with the beginning of IR flares (Eikenberry et al. 1998). The time delay of 200-400 s reported by them between the X-ray and IR peaks is probably due to the time required for the evolution of the IR flare as well as for the readjustment of the accretion disk. Our calculated time delay of $804 \mathrm{~s}$ is in good agreement with the measured time delay between $15 \mathrm{GHz}$ and $8.3 \mathrm{GHz}$ observations reported by M98 (MJD 50583). In a detailed study of X-ray - radio observations in GRS 1915+105, Klein-Wolt et al. (2002) measure the time delay of 40-45 min from the beginning of state $\mathrm{C}$ to the peak radio emission at $15 \mathrm{GHz}$, which agrees with our estimate of $43 \mathrm{~min}$. The observations of M98 (MJD 50700) has measured time delay of $\sim 960$ s between IR and $8.3 \mathrm{GHz}$, while our calculated value is $3351 \mathrm{~s}$ $(0.93 \mathrm{~h})$, the difference is about $40 \mathrm{~min}$. Inspection of Fig. 2 of M98 show that a previous IR peak is separated by about the same amount of time. This suggests that the $8.3 \mathrm{GHz}$ flare most likely corresponds to the IR flare $\sim 0.9 \mathrm{~h}$ prior to the $8.3 \mathrm{GHz}$ peak. For observation on MJD 50705, the discrepancy of $547 \mathrm{~s}$ between the measured and the calculated time delay between IR and radio emission could be due to larger uncertainty in the measured time delay because of the presence of two IR peaks. From the radio observations at 4.8 and $8.6 \mathrm{GHz}$ on MJD 51751, Fender et al. (2002) find a delay time of $\sim 600 \mathrm{~s}$ based on a broad peak $(\sim 300 \mathrm{~s})$ in the cross correlation function while our estimate of time delay is $1030 \mathrm{~s}$. Although the exact answer to this discrepancy is not clear, it may be due to uncertainty in the time delay measurements.
Table 2. Calculated time delays at different frequencies for the multiwavelength data from literature.

\begin{tabular}{cccccc}
\hline \hline MJD & $\begin{array}{c}\nu_{1} \\
(\mathrm{~Hz})\end{array}$ & $\begin{array}{c}\nu_{2} \\
(\mathrm{~Hz})\end{array}$ & $\begin{array}{c}\Delta t \\
(\mathrm{obs})\end{array}$ & $\begin{array}{c}\Delta t \\
(\mathrm{calc})\end{array}$ & Ref. \\
\hline 50675 & $\mathrm{X}$-ray & $1.4 \times 10^{14}$ & $200-400$ & 37 & E98 \\
50583 & $1.5 \times 10^{10}$ & $8.3 \times 10^{9}$ & $\sim 750$ & 804 & M98 \\
50698 & X-ray & $10^{15}$ & $2400-2700^{*}$ & 2584 & K02 \\
50700 & $1.4 \times 10^{14}$ & $8.3 \times 10^{9}$ & $\sim 960$ & 3351 & M98 \\
50705 & $1.4 \times 10^{14}$ & $1.5 \times 10^{10}$ & $\sim 2000$ & 2547 & F98 \\
51751 & $8.6 \times 10^{9}$ & $4.8 \times 10^{9}$ & $\sim 600$ & 1030 & F02 \\
\hline
\end{tabular}

${ }^{*}$ When calculated from the beginning of State C.

It may be noted here that the time delays estimated using our simple model of synchrotron bubble are in good agreement with many of the observed values between different frequencies available in the literature. However, naive application of this model predicts much higher flux densities than observed at higher frequencies. This discrepancy may be the subject of future studies.

Acknowledgements. We thank the referee, Dr. G. G. Pooley for meticulously reading the paper and making important comments. We also thank several of our colleagues, especially Dr. A. K. Jain and Prof. A. R. Rao for stimulating discussions. We thank the staff of the GMRT that made these observations possible. GMRT is run by the National Center of Astrophysics of the Tata Institute of Fundamental Research. This research has made use of data obtained from the High Energy Astrophysics Science Archive Center (HEASARC), provided by NASA's Goddard Space Flight Center and also of NASA's Astrophysics Data System Abstract Service.

\section{References}

Castro-Tirado, A. J., Brandt, S., \& Lund, N. 1992, IAUC, 5590 Dhawan, V., Mirabel, I. F., \& Rodríguez, L. F. 2000, ApJ, 543, 373

Eikenberry, S. S., Matthews, K., Morgan, E. H., Remillard, R. A., \& Nelson, R. W. 1998, ApJ, 494, L61 (E98)

Fender, R. P., \& Pooley, G. G. 1998, MNRAS, 300, 573 (F98)

Fender, R. P., Garrington, S. T., McKay, D. J., et al. 1999, MNRAS, 304, 865

Fender, R. P., Rayner, D., Trushkin, S. A., et al. 2002, MNRAS, 330, 212 (F02)

Feroci, M., Matt, G., Pooley, G., et al. 1999, A\&A, 351, 985

Greiner, J., Cuby, J. G., \& McCaughrean, M. J. 2001, Nature, 414,522

Harmon, B. A., Deal, K. J., Paciesas, W. S., et al. 1997, ApJ, 477, L85

Hjellming, R. M., \& Johnston, K. J. 1988, ApJ, 328, 600

Klein-Wolt, M., Fender, R. P., Pooley, G., et al. 2002, MNRAS, 331, 745 (K02)

Mirabel, I. F., \& Rodríguez, L. F. 1994, Nature, 371, 46

Mirabel, I. F., Dhawan, V., Chaty, S., et al. 1998, A\&A, 330, L9 (M98)

Muno, M. P., Remillard, R. A., Morgan, E. H., et al. 2001, ApJ, 556, 515

Pooley, G. G., \& Fender, R. P. 1997, MNRAS, 292, 925

Swarup, G., Ananthakrishnan, S., Kapahi, V. K., et al. 1991, Curr. Sci., 60, 95

van der Laan, H. 1966, Nature, 211, 1131

Yadav, J. S. 2001, ApJ, 548, 876 